\title{
Ultracompact alignment-free single molecule fluorescence device with a foldable light path
}

\author{
Niraj Kumar Singh, ${ }^{a}$ Jenu V. Chacko, ${ }^{a}$ Varun K. A. Sreenivasan, ${ }^{\mathrm{b}}$ Suman Nag, ${ }^{\mathrm{a}}$ and Sudipta Maitia \\ ${ }^{a}$ Tata Institute of Fundamental Research, Homi Bhaba Road, Colaba, Mumbai 400005, India \\ ${ }^{\text {b} M a c q u a r i e ~ U n i v e r s i t y, ~ M Q ~ P h o t o n i c s ~ C e n t r e, ~ D e p a r t m e n t ~ o f ~ P h y s i c s ~ a n d ~ A s t r o n o m y, ~ S y d n e y ~ 2109, ~ A u s t r a l i a ~}$
}

\begin{abstract}
Instruments with single-molecule level detection capabilities can potentially benefit a wide variety of fields, including medical diagnostics. However, the size, cost, and complexity of such devices have prevented their widespread use outside sophisticated research laboratories. Fiber-only devices have recently been suggested as smaller and simpler alternatives, but thus far, they have lacked the resolution and sensitivity of a full-fledged system, and accurate alignment remains a critical requirement. Here we show that through-space reciprocal optical coupling between a fiber and a microscope objective, combined with wavelength division multiplexing in optical fibers, allows a drastic reduction of the size and complexity of such an instrument while retaining its resolution. We demonstrate a $4 \times 4 \times 18 \mathrm{~cm}^{3}$ sized fluorescence correlation spectrometer, which requires no alignment, can analyze kinetics at the single-molecule level, and has an optical resolution similar to that of much larger microscope based devices. The sensitivity can also be similar in principle, though in practice it is limited by the large background fluorescence of the commonly available optical fibers. We propose this as a portable and field deployable single molecule device with practical diagnostic applications. @ 2011 Society of Photo-Optical Instrumentation Engineers (SPIE). [DOl: 10.1117/1.3548311]
\end{abstract}

Keywords: fluorescence correlation spectroscopy; fiber-based fluorescence correlation spectroscopy; compact fluorescence correlation spectroscopy; fiber confocal.

Paper 10667R received Dec. 20, 2010; revised manuscript received Jan. 4, 2011; accepted for publication Jan. 4, 2011; published online Feb. 25, 2011.

\section{Introduction}

Fluorescence correlation spectroscopy (FCS) is a powerful single-molecule level technique that can measure the hydrodynamic size of molecules and chemical kinetic rate constants. ${ }^{1,2}$ Since the size of a fluorescent ligand changes when it attaches to an acceptor molecule, this technique has obvious possibilities in disease diagnostics, promising rapid and quantitative determination of binding and aggregation in the solution phase. Its high sensitivity and microscopic probe volumes imply that miniscule amounts (often in the femto-mole range) of sample and reagents are needed for an experiment. FCS hardware can also be easily adapted to make other types of fluorescence measurement simultaneously, such as the determination of fluorescence lifetime through Time Correlated Single Photon Counting. However, the spread of this technology beyond the optics laboratory has been hampered by the large size and cost of the available instruments. In addition, the complicated optical and electronic machinery of a typical FCS instrument lacks the robustness required for "outof-the-laboratory" situations.

This scenario can completely change if an FCS instrument is designed such that it is compact and foldable, is impervious to misalignments, and is affordable, yet sensitive. The first step in this direction was taken when we introduced the fully fiber-optic FCS device. ${ }^{3}$ It used the same single-mode fiber for both excitation and collection of fluorescence. The tip of the fiber served both as a "focusing lens" and as the confocal aperture. ${ }^{4}$ However, the resolution and the sensitivity were poor due to the low

Address all correspondence to: Sudipta Maiti, Tata Institute of Fundamental Research, Homi Bhaba Road, Colaba, Mumbai 400005, India. Tel: +91 2222782716; Fax: + 912222804610 ; E-mail: maiti@tifr.res.in. numerical aperture (NA) of the single-mode fiber. While this is still useful for monitoring multiply-labeled objects such as protein aggregates, ${ }^{5}$ the device is impractical for single molecule detection. In addition, the instrument still required alignment of the excitation laser into the fiber, and of the fluorescence signal into the small aperture of the typical avalanche photodiode detector. Subsequent work by others, e.g., incorporating a microsphere lens at the end of the fiber, ${ }^{6}$ have now taken the sensitivity down to the single molecule levels. However, it is still not comparable in resolution and sensitivity to a full-frame instrument, which employs a high NA objective, and it still requires sensitive alignment of lasers and fluorescence beams into small apertures.

We present a strategy that combines a conventional high NA objective with the fiber scheme. It retains the compactness of the fiber scheme, yet promises resolution and sensitivity similar to that obtained from the full-frame devices. In addition, it uses fiber pigtailed lasers and fiber-based splitters/combiners incorporating specific wavelength-selective optics (i.e., wavelength division multiplexing), to eliminate any need of alignment.

\section{Methods}

In FCS, both the excitation and the emission paths need to be precisely defined, with respect to the optical axes of the instrument, and this is typically achieved through a series of lenses and mirrors. This approach adds bulk to the typical FCS instrument and makes it optically complex. However, any alternative

$1083-3668 / 2011 / 16(2) / 025004 / 4 / \$ 25.00$ @ 2011 SPIE 
scheme that proposes to make FCS alignment-free and robust must do so while satisfying these requirements.

There are two primary requirements for the excitation path. The laser beam has to be enlarged to an appropriate size in order to fill the back aperture of the objective lens (if high spatial resolution is required) and collimated. Furthermore, the collimated beam has to be steered along the optical axis of the microscope objective to the specimen. ${ }^{7}$ In the emission path, the back-propagating fluorescence beam has to be separated from the excitation beam by a dichroic filter and focused by a "tube lens" (which has a typical focal length of 15 to $20 \mathrm{~cm}$ ) to a small confocal aperture (typically a few tens of micrometers) in front of the detector. This is typically achieved by moving either the aperture or the fluorescence spot in three dimensions (e.g., by moving the tube lens). A tube lens with a smaller focal length may reduce the size of the instrument, but that will also make the aperture size (required for confocality) proportionally smaller, demanding even more precise alignment.

In the scheme presented here [Fig. 1(a)], we use a single mode fiber for carrying the excitation laser beam to the objective lens. The fiber is fixed connection (FC) connectorized, and a plate holding an FC receptacle (F1) is mounted at the base of a small rigid cage assembly. This assembly holds the fiber, a tube lens (L1), a microscope objective (O1), and a sample holder (S1). The fiber has a NA of 0.11 and a divergence of about 0.11 rad. The tube lens ( $\mathrm{L} 1$, of focal length $3.5 \mathrm{~cm}$ ) is placed 3.5 $\mathrm{cm}$ above the fiber tip, so that the excitation light is collimated after the lens and has a size of about $1 \mathrm{~cm}$. Immediately above this lens, we mount a 1.2 NA water-immersion $60 \times$ objective (O1, Olympus, Japan, Model Number UPLSAPO). O1 focuses the light onto the specimen placed on a cover glass, which is mounted on another plate in the cage assembly (S1). S1 has a circular hole in the middle that allows the light to pass through and accommodates the nose-piece of the objective. The fiber at the other end is pigtailed to the source laser (Oz optics, Canada, Model Number OZ-2000-532-3.5/125-S-40-2 A-0.5-1 O-bl), eliminating any need for alignment there.

The fluorescence emission from the specimen is omnidirectional, and given the reciprocal nature of light propagation, the portion collected by the objective lens is automatically focused back onto the tip of the excitation fiber. The small focal length of the tube lens helps to make the assembly small, but it does not create alignment issues. An actual photograph of this optical unit showing its size is shown in Fig. 1(b). The fiber tip acts as the confocal aperture, similar to the strategy employed earlier ${ }^{3}$ for limiting the observation volume. It is also similar to the approach used previously in fiber-based confocal microscopes. ${ }^{3,6,8-13}$ The fiber is single mode for the emission wavelength, so the aperture size is optimal.

This fiber carries the fluorescence back towards the source laser. However, between the laser end and the objective end $(\mathrm{OE})$ of the fiber, we place a small wavelength division multiplexer unit [detailed schematic in Fig. 1(c)]. This contains a dichroic mirror (DM1) which reflects the excitation beam after it is collimated by a lens (L1). This collimated beam is further focused onto a fiber at the OE using a second lens (L2). The fluorescence from the sample is carried back through the same fiber onto the dichroic mirror (DM1), which transmits it toward the output port of the multiplexer and a lens (L3) focuses this onto a multimode fiber. A filter (F1) is placed before the multimode

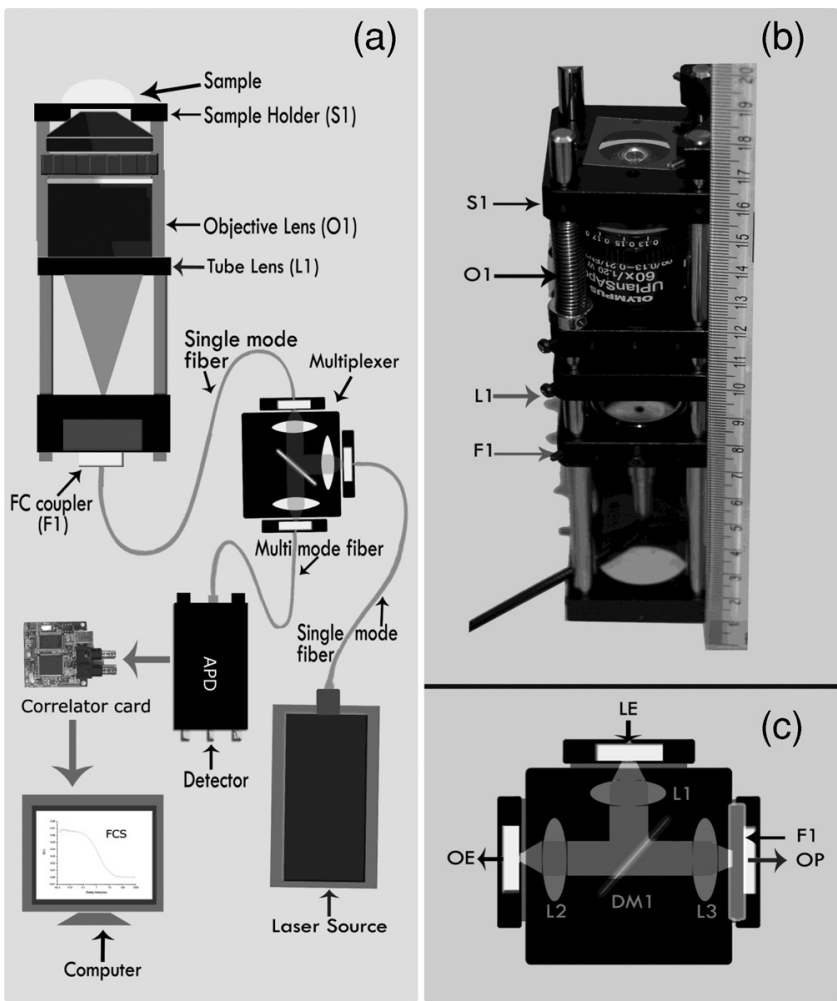

Fig. 1 (a) Schematic of the set up showing the optical unit containing the objective (O1) and the tube lens (L1), and sample and fiber holder plates (S1 and F1, respectively). The figure also shows fiberpigtailed laser source, fiber-multiplexing assembly containing dichroics and filters, the avalanche photodiode detector, the correlator, and the computer. (b) Actual photograph of the optical unit showing its size. (c) Details of the wavelength division multiplexer unit containing a dichroic (DM1), lenses to collimate the light beam at the dichroic (L1, L2, and L3), and a band-pass filter (F1) at the transmission port.

fiber for rejecting any remaining excitation light in the beam. This fiber carries the fluorescence to a fiber-coupled avalanche photodiode detector (SPCM-CD-2882, Perkin Elmer Optoelectronics, Canada). The whole fiber assembly is custom manufactured to our specifications by Oz Optics, Canada. The detector sends electrical outputs to a small USB connectorized processor card (Correlator.com, NJ, Model Number FLEX99OEM-12D) which interfaces with a small laptop computer and computes the autocorrelation function. The software for the acquisition and data-logging is written in-house using LabView (National Instruments, USA). A comparison of the current schematic with that of a conventional FCS set up is presented in Fig. 2. In a typical FCS set up, the optical path ABCDE from the laser source up to the dichroic mirror (DM1) involves four mirrors M1, M2, M3, M4 and two lenses L1 and L2 [Fig. 2(a)]. In the fiber-based FCS system, this is replaced by the optical path A1B1 using a single mode fiber (SMF1) with a core diameter of $3.5 \mu \mathrm{m}$ [Fig. 2(b)]. In the conventional set up, the excitation beam expansion and collimation is performed using lens L1 and L2, before it reaches the objective lens (O1) which then focuses the excitation onto the sample [Fig. 2(a)]. In the fiber-based system, a single wavelength division multiplexer unit (WDM) replaces this entire lens assembly. This has a dichroic mirror (DM1) and three lenses [see Fig. 1(c)] integrated within combination with 
(a)

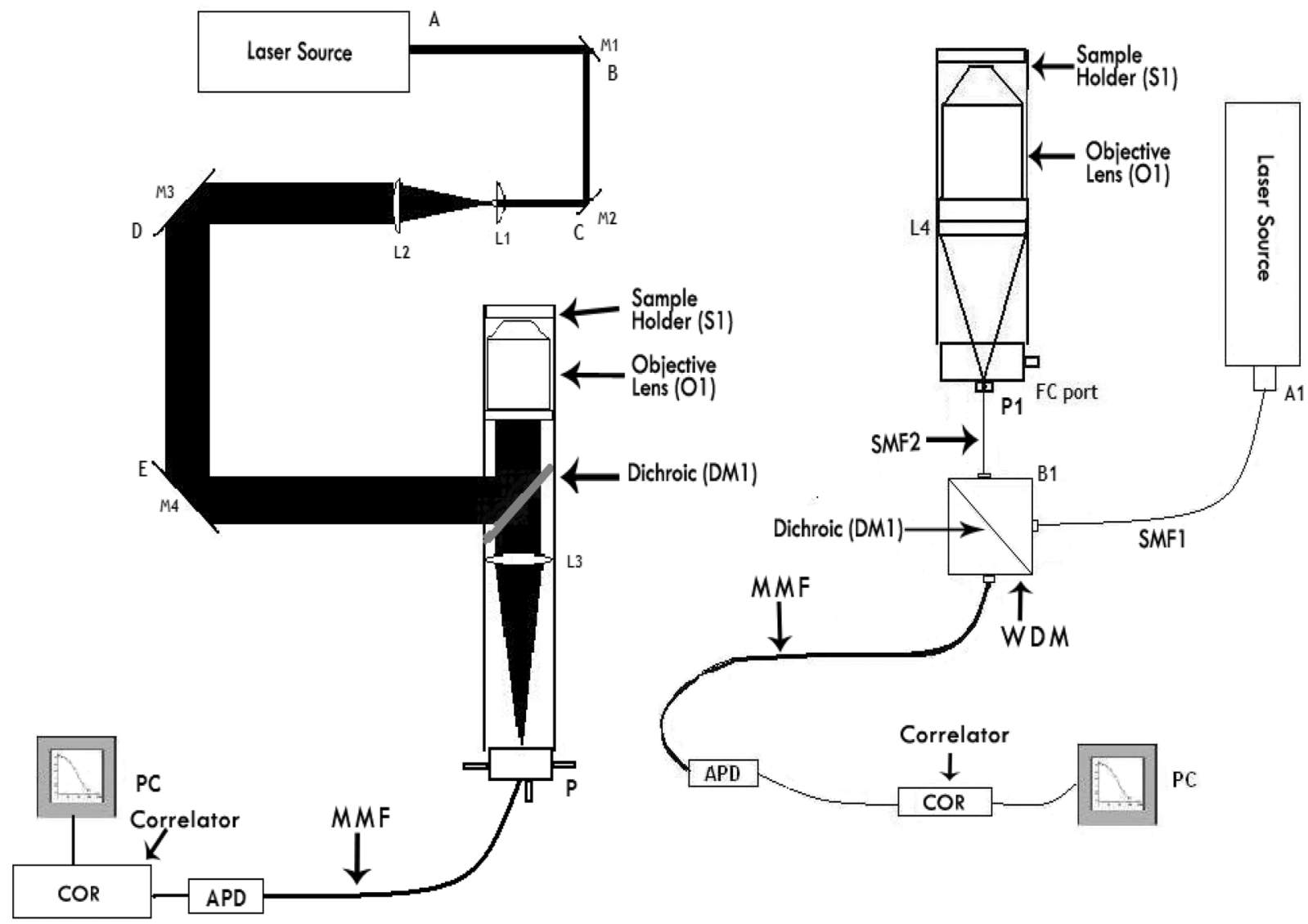

(b) 
Singh et al.: Ultracompact alignment-free single molecule fluorescence device...

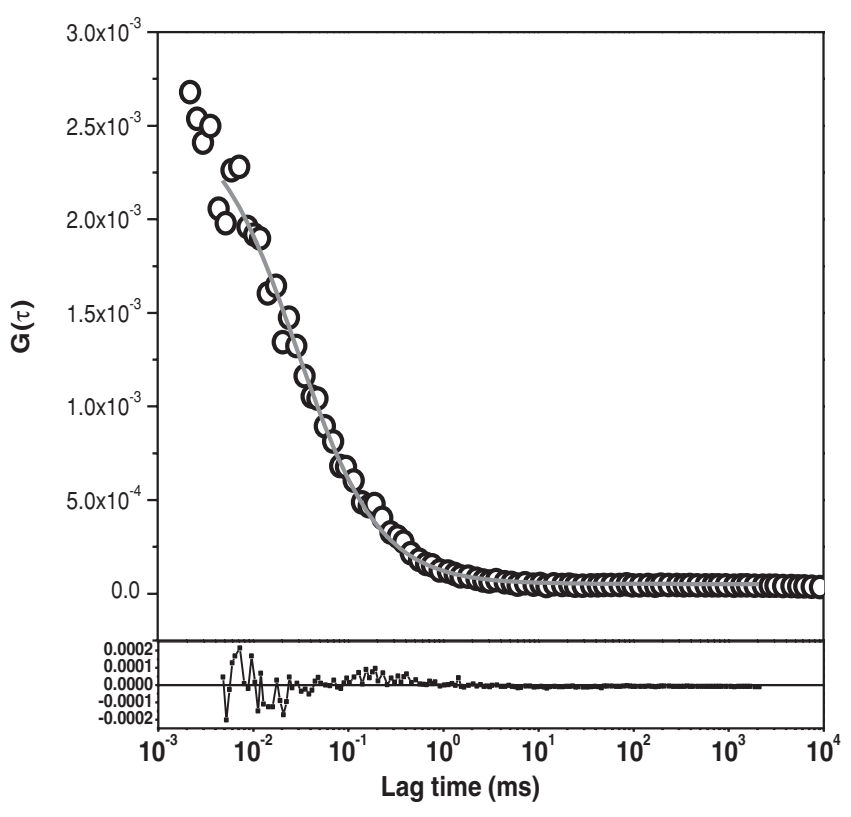

Fig. 3 Autocorrelation data (open circles) and a single component fit (gray solid line) obtained from a $470 \mathrm{nM}$ rhodamine B solution. The corresponding residual plot is shown at the bottom (black).

The most notable feature of the instrument is the size of the optical unit [Fig. 1(b)]. At $4 \times 4 \times 18 \mathrm{~cm}^{3}$, it occupies about $1 \%$ of the volume of a typical FCS instrument. Other electronic units and the laser, etc., are also quite small and can easily fit inside a separate palm-sized box, which is connected to the optical unit through a single optical fiber. The other notable feature of the instrument is that the light path is fiber-based and foldable, and does not require any alignment [Fig. 1(a)]. The only open part of the path is between the fiber and the objective, but alignment here is automatic, and is impervious to small changes in the relative positions of the optical elements. The instrument is therefore expected to be robust and field-deployable. We note that the instrument can be used for Photon Count Histogram and/or other modalities of fluorescence data analysis which demand single molecule level sensitivity. In addition, if a pulsed diode laser replaces the continuous wave laser and a high time resolution avalanche photodiode is used as a detector, fluorescence lifetime determination can also be performed simultaneously. We hope that this instrument will pave the way for making single molecule fluorescence technology available as a point-of-care clinical diagnostic tool.

\section{References}

1. D. Magde, W. W. Webb, and E. Elson, "Thermodynamic Fluctuations in a Reacting System - Measurement by Fluorescence Correlation Spectroscopy," Phys. Rev. Lett. 29(11), 705-708 (1972).

2. S. Maiti, U. Haupts, and W. W. Webb, "Fluorescence correlation spectroscopy: Diagnostics for sparse molecules," Proc. Natl. Acad. Sci. U.S.A. 94(22), 11753-11757 (1997).

3. K. Garai, M. Muralidhar, and S. Maiti, "Fiber-optic fluorescence correlation spectrometer," Appl. Opt. 45(28), 7538-7542 (2006).

4. J. Balaji, K. Garai, S. Chakrabarti, and S. Maiti, "Axial resolution limit of a fiber-optic fluorescence probe," Appl. Opt. 42(19), 3780-3784 (2003).

5. K. Garai, R. Sureka, and S. Maiti, "Detecting amyloid-beta aggregation with fiber-based fluorescence correlation spectroscopy," Biophys. J. 92(7), L55-L57 (2007).

6. H. Aouani, F. Deiss, J. Wenger, P. Ferrand, N. Sojic, and H. Rigneault, "Optical-fiber-microsphere for remote fluorescence correlation spectroscopy," Opt. Express 17(21), 19085-19092 (2009).

7. P. Sengupta, J. Balaji, and S. Maiti, "Measuring diffusion in cell membranes by fluorescence correlation spectroscopy," Methods 27(4), 374387 (2002).

8. D. Dickensheets and G. S. Kino, "Scanned optical fiber confocal microscope.," Proc. SPIE 2184, 39-47 (1994).

9. L. Giniunas, R. Juskaitis, and S. V. Shatalin, "Scanning Fiberoptic Microscope," Electron. Lett. 27(9), 724-726 (1991).

10. T. Ota, H. Fukuyama, Y. Ishihara, H. Tanaka, and T. Takamatsu, "In situ fluorescence imaging of organs through compact scanning head for confocal laser microscopy," J. Biomed. Opt. 10(2), 024010 (2005).

11. M. R. Harris, "Scanning confocal microscope including a single fibre for transmitting light to and receiving light from an object "U. S. Patent No. 5,120,953 (1992).

12. J. B. Pawley, "Biological Confocal Microscopy," 3rd ed., pp. 508-515, Springer, New York (2006).

13. B. A. Flusberg, E. D. Cocker, W. Piyawattanametha, J. C. Jung, E. L. M. Cheung, and M. J. Schnitzer, "Fiber-optic fluorescence imaging," Nat. Methods 2(12), 941-950 (2005).

14. C. T. Culbertson, S. C. Jacobson and J.M. Ramsey, "Diffusion coefficient measurements in microfluidic devices," Talanta 56(2), 365-373 (2002). 